\title{
IMPROVING THE PROTECTION OF EHV TEED FEEDERS USING LOCAL AGENTS
}

\section{V Coury ${ }^{1}$}

\author{
${ }^{1}$ University of São Paulo, Brazil \\ ${ }^{2}$ Cornell University, USA
}

J S Thorp ${ }^{2}$

\section{INTRODUCTION}

The protection of multi-terminal lines is not as simple as that of two-terminal lines. They usually experience additional problems caused by the intermediate infeed from the third terminal, an outfeed, different line lengths to the tee point, etc. The limitations of non-pilot distance relays for their protection have been acknowledged for decades. These limitations lead to the traditional distance relaying approach to experience severe underreach and overreach problems. The relay first zone reach may not even extend beyond the tee point in some underreach cases (1),(2).

An approach based on adaptive relaying to face such a situation and some fundamental concepts concerning it was presented by Rockefeller (3). Horowitz et al (4) define adaptive relaying as a protection philosophy which permits and seeks to make adjustments to various protection functions in order to make them more attuned to prevailing power system conditions. An adaptive setting concept for two and three terminal lines, which can respond to changes in the network conditions, was proposed by Xia et al (5), (6). Stedall et al (7) also proposed an investigation into the use of adaptive setting techniques for improved distance back-up protection.

This paper discusses the adaptation of the settings of distance relays for multi-terminal lines employing agents. Agents are software processes capable of searching for information in networks, interacting with pieces of equipment and performing tasks on behalf of their owners (relays). Moreover, they are autonomous and cooperative. Very few publications concerning the application of agents in the protection field have been reported in the literature. Tomita et al (8) proposed a cooperative protection system utilizing agents. Relay agents were constructed, and the cooperation of the main agents was simulated for primary, backup and adaptive protection.

Results illustrating the improved performance of the adaptive method proposed compared to conventional fixed settings are presented in the following sections.

\section{AGENT TECHNOLOGY}

A software agent is a computer program that takes independent action based on events in the surrounding environment (Genesereth (9)). Under the control of some application that owns the agent, it is sent forth or placed at some site within a network where it takes local actions on behalf of its owners. Agents are dynamic entities. For example, an agent can exhibit dynamically
K M Hopkinson ${ }^{2}$

K P Birman ${ }^{2}$ changing behavior, receiving updated parameter settings from the owner or from other agents participating in the same application. An agent can also learn from the environment; the term "intelligent" is sometimes applied to such agents, particularly if the learning method involves artificial intelligence tools, or neural networks. Agents can be further subdivided based on their mobility. Mobile agents can literally travel from one location to another while maintaining their state and functionality. One imagines such an agent as moving through a network in search of certain data, or for locations having certain attributes. In contrast, local agents are placed by the owner and then remain in the same location throughout their computational lives. Both kinds of agents normally have the ability to communicate with one another making mobility a lesser benefit than it might appear at first glance.

In the present paper, we consider the simplest kinds of agents: local, non-intelligent ones. Our work seeks to demonstrate advantages stemming from the rapid response time made possible by placing a computational task close to the point where an action or measurement is needed, and the simplicity of the power systems architecture afforded by the ability to send code to general purpose computing platforms. The basic idea is that by doing so, new control paradigms can be introduced and executed efficiently, without changing the basic computing architecture of the power systems network.

The use of agents raises security considerations that go beyond those encountered in traditional software systems. Among the issues that need to be considered are the mechanism for installing agents and for communicating parameter updates to them, the means by which a computing platform or agent authenticates incoming messages, and the enforcement of agent resource restrictions, such as the use of scarce computational cycles or space in databases and file systems. Prior to deploying agents in mission-critical settings, one would need satisfying answers on all of these fronts. Preliminary work towards policies addressing these issues can be found in (10).

\section{THE PROPOSED COMMUNICATION STRUCTURE AND THE POWER SYSTEM STUDIED}

\section{The communication structure (middleware)}

The computing infrastructure in which our agent community will operate is a subject of active speculation at the time of this writing. The existing power grid 
communication infrastructure is composed of a hodgepodge of equipment ranging from high-end fiber optic cables with large data bandwidth to crude systems with limited data transfer capabilities to nothing at all. Few utility communications lines are interconnected. However, this picture will change as utilities deploy ever more elaborate control mechanisms, demanding increasingly rich communication frameworks, steps that can easily be foreseen as part of the battle to improve operating efficiencies.

The relay scheme proposed here has modest network resource requirements compared to what we believe will be available in the near future. It is expected that the need for greater communication will result in a Utility Intranet (UI) that would interconnect control areas and their dependent entities using a relatively high capacity network similar to the Internet but physically separate from it and dedicated to the power systems application. However, we caution that predictions of network bandwidth requirements and total network capacity can be deceptively reassuring. History has shown a tendency for networked applications to use all bandwidth made available to them.

To evaluate the feasibility of employing agents and network communication in power-systems relay protection, we need to understand the reliability and latency requirements of the relays. By doing so, we can determine whether or not a proposed combination of network hardware and communication protocols can meet the protection system's needs. Relays play a critical role in power grid operations with low tolerance for failures. One can identify that situations in communication between agents used for relay control might need to take place in as little as $10 \mathrm{~ms}$, a latency realm leaving little time to recover from data transmission failures. It is therefore critical to ask whether available technology can meet those requirements.

In order to face some of the network limitations, communication protocols like Cornell's Spinglass have been developed by Birman et al (11). Spinglass allows the user to achieve high levels of reliability using protocols that probabilistically mask latency fluctuations and message loss. Spinglass could be optimized for use in power relaying and might form a powerful infrastructure for improved protection schemes.

\section{The three-ended system utilized}

In order to simulate the real system encountered in practice, this work makes use of a digital simulator of faulted transmission lines known as PSCAD/EMTDC (Electromagnetic Transients and Controls Simulation Engine) (12). It should be mentioned that although the technique described is based on Computer Aided Design (CAD), practical considerations such as the Capacitor Voltage Transformer (CVT), Current Transformer (CT) and anti-aliasing filters on system fault data were also included in the simulation.

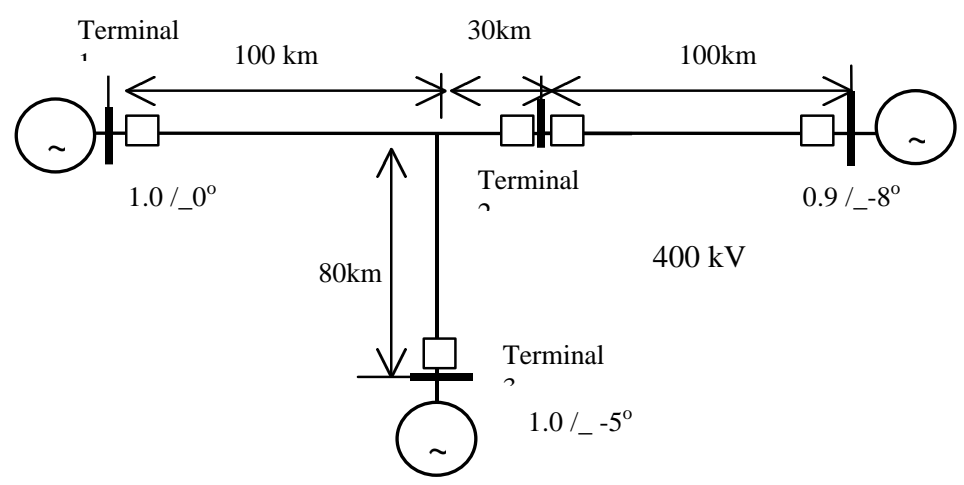

Figure 1 The three-ended system utilized for tests

A $400 \mathrm{kV}$ system with tee configuration is shown in Fig. 1. The different line lengths are presented in the figure, as well as the voltage levels at the busbars. This work focuses on line-to-ground faults, since most faults in power system transmission lines are of this type.

The protection system associated with this configuration was simulated for conventional non-pilot protection as well as a portion of the agent based relaying approach proposed. Digital distance relays with three zone quadrilateral characteristics located at terminals 1, 2 and 3 were simulated. DFT (Discrete Fourier Transform) routines were utilized in order to filter the faulted waves. Circuit breakers were associated with the relays in order to isolate the line in case of an internal fault.

\section{AGENT RELAYS}

Fig.2 presents the agent mechanism for protection purposes. It can be initially divided into an adaptive prefault approach and a post-fault transfer trip action. The mechanism utilizes local agents at terminals 1,2 and 3 communicating with each other.

\section{Adaptive Pre-fault Approach}

The main purpose of the proposed agent mechanism is to carry out the adaptation of the settings of distance relays for multi-terminal lines in order to ensure correct performance over a wide variety of operation conditions.

Initially, local agents for terminals 1,2 and 3 can be defined as:

Operation Agent: This is a local agent, which monitors the operating condition at a terminal. The following parameters related to the system condition must be obtained from on-line measurements and/or calculations:

- Pre-fault busbar voltages

- Power transfer angles

- Source impedances

Breaker Status Agent: This local agent monitors changes in the system topology based on circuit breaker status. 
The two local agents, shown in Fig.2, must communicate with the Coordination Agent, as defined in item 3.

Coordination Agent: The coordination agent collects information, makes decision, and disseminates information to the other two Coordination agents. The Coordination Agent at each terminal can then choose the correct relay characteristic, based on the information received.

We propose that a group of settings be calculated offline, and that these parameters be renewed from time to time as the system condition changes beyond a certain limit, based on the Coordination Agent information. Then, when a fault occurs the relay will operate close to its ideal characteristic.

\section{First Zone Transfer Trip}

This action takes place after the fault occurrence. In order to decrease the clearance time, when a fault is detected in the first zone of any of the relays located at the three terminals, a trip signal is sent through the communication network to the other two ends. A First Zone Local Agent coordinates the first zone transfer trip.

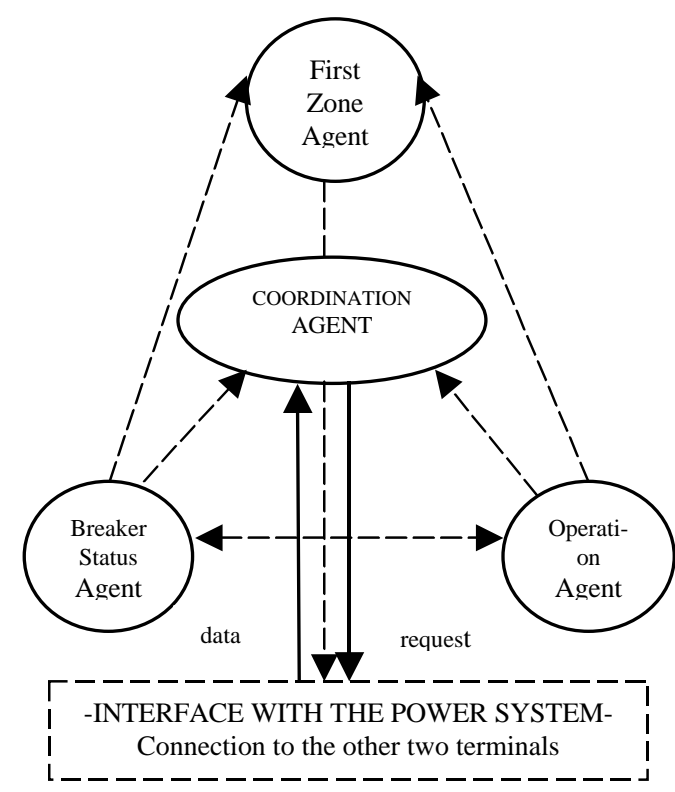

Figure 2 Agent mechanism in one terminal for protection purpose

\section{RESULTS OBTAINED UTILIZING AGENT TECHNOLOGY}

\section{Adaptive Pre-fault Approach Results}

Distance relaying techniques have attracted considerable attention for the protection of transmission lines. The principle of this technique measures the impedance at a fundamental frequency between the relay location and the fault point, thus determining if a fault is internal or external to a protection zone. The relationship between voltage and current is used for this purpose. This relationship is disturbed when there is infeed or outfeed. As mentioned earlier, this can make the relay experience severe underreach or overreach problems, especially considering non-pilot protection schemes for three terminal lines. Another situation that has to be taken into account is when the tap is not working for some reason. The setting of distance relays must reflect all the situations described earlier. Fig. 3(a) shows the typical non-pilot distance protection with the first zone for a three terminal line at the condition represented in Fig. 1. As shown in the figure, only approximately 20 from 210 $\mathrm{km}$ obtained instantaneous trip from the three ends of the lines in the case of an internal fault. Fig. 3(b) illustrates the improvement that can be found with the adaptive scheme utilizing Agents that is proposed in this work. The instantaneous trip area increased to approximately $67 \mathrm{~km}$ (more than three times the nonpilot condition) for the same situation. As mentioned before, the proposed adaptive scheme can alter settings to ensure better performance over a wide variety of operation conditions.

\section{Clearance Time}

In order to highlight the efficiency of the proposed scheme, this section presents a figure comparing the clearance time for the conventional non-pilot protection as well as for the proposed scheme utilizing Agents.

Fig. 4 presents the clearance time for the three-ended line shown in Fig. 1. Line-to-ground faults are applied to different locations on leg 1-T, considering different fault inception angles and fault resistance of $10 \mathrm{ohms}$. The figure shows the average clearance time considering legs 1,2 and 3 for the non-pilot protection as well as the proposed scheme considering three different traffic conditions. A considerable decrease in clearance time considering the proposed scheme can be observed.

It should be noted that an opening time of approximately three cycles for the circuit breaker utilized was considered. It isolates the fault by interrupting the current at or near a current zero. A coordination delay for zone two of the order of $0.3 \mathrm{~s}$ was also utilized.

\section{CONCLUSION}

An application of agents acting within a communication structure to adaptive relay settings for multi-terminal lines was presented in this paper. Results illustrating the performance of the new method proposed compared to conventional fixed settings were shown. The new approach based on agents was divided in an adaptive pre-fault approach and a post-fault transfer trip action. In order to test the proposed scheme, a digital simulation of a faulted transmission line was performed utilizing the PSCAD/EMTDC software. The protection system associated with this configuration was also simulated both for conventional non-pilot protection as well as for the Agent based relaying. 


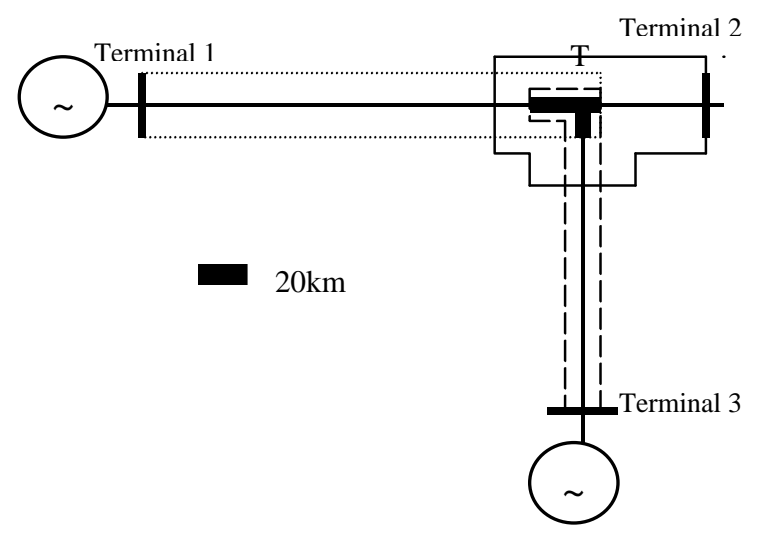

(a) Non-pilot protection

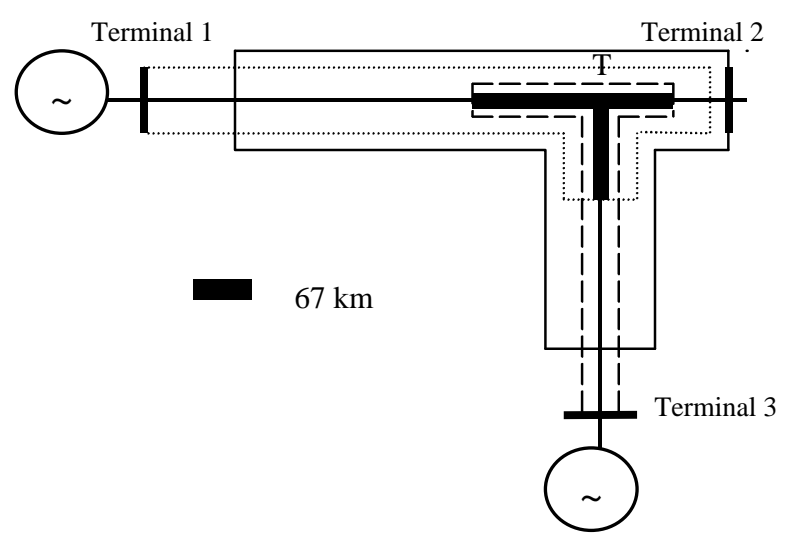

(b)Agent Technology proposed - Adaptive pre-fault scheme

Figure 3 Distance relaying of a three ended transmission line

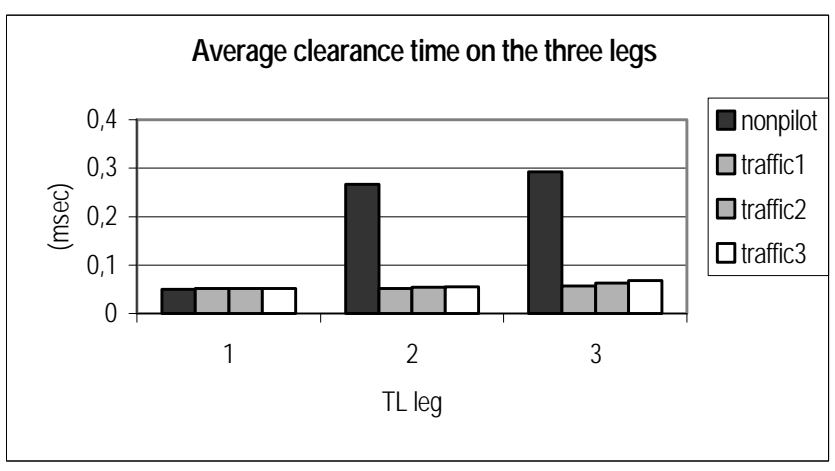

Figure 4 Average clearance time for the three legs considering non-pilot protection and the scheme proposed

The results showed that the new approach would alter adaptive settings to ensure correct performance over a wide variety of operation conditions, which is an improvement compared to fixed settings for the relays. Moreover, a considerable decrease in clearance time compared to conventional non-pilot protection was observed.

It should then be emphasized that the new approach is not only a solution for a well-known problem, but also a very attractive alternative to improve the performance of protection systems.

\section{ACKNOWLEDGMENTS}

The authors acknowledge the research support by subcontract No. 35352-6085 with Cornell University under WO 8333-04 from the Electric Power Institute and the U.S. Army Research Office. Our acknowledgments also extend to DARPA/RADC grant F30602-99-1-6532 as well as FAPESP (Fundação de Amparo à Pesquisa do Estado de São Paulo -Brazil).

\section{REFERENCES}

(1) Horowitz S H and Phadke AG, 1996 "Power System Relaying", John Wiley and Sons.

(2) IEEE Working Group, 1979, "Protection Aspects of Multi-terminal Lines", IEEE Special Publication, 79 TH0056-2-PWR.

(3) Rockefeller G D, Wagner C L, Linders J R, Hicks K L and Rizy D T, 1988 “Adaptive Transmission Relaying Concepts for Improved Performance”, IEEE Trans. on Power Delivery, 3 (4), 1446-58.

(4) Horowitz S H, Phadke A G and Thorp J S, 1988 “Adaptive Transmission System Relaying”, IEEE Trans. on Power Delivery, 3 (4), 1436-45.

(5) Xia Y Q, Li K K and David A K , 1994 "Adaptive Relay Setting for Stand Alone Digital Distance Protection", IEEE Trans. on Power Delivery, 9(1), 48091.

(6) Xia Y Q, David A K and Li K K, 1994 "HighResistance Faults on a Multi-Terminal Line: Analysis, Simulated Studies and an Adaptive Distance Relaying Scheme", IEEE Trans. on Power Delivery, 9 (1), 492500.

(7) Stedall B, Moore P, Johns A, Goody J and Burt M, 1996 "An Investigation into the use of Adaptive Setting Techniques for Improved Distance Back-up Protection", IEEE Trans. on Power Delivery, 11 (2), 757- 62.

(8) Tomita Y, Fukui C, Kudo H, Koda J, Yabe K, 1998 "A Cooperative Protection System with an Agent Model", IEEE Trans. on Power Delivery, 13 (4), 106066.

(9) Genesereth M and Ketchpel S, 1994 "Software Agents", Communications of the ACM, 37(7), 48-52, 147.

(10) Schneider F, 1997 "Towards Fault-tolerant and Secure Agentry", $111^{\text {th }}$ International Workshop on Distributed Algorithms, 1320, September 1-14.

(11) Birman K, Hayden M, Ozkasap O, Xiao Z, Budiu M and Minsky Y, 1999 "Bimodal Multicast", ACM Trans. on Computer Systems, 17(2).

(12) PSCAD/EMTDC Manual, 1998 Getting Started, Manitoba HVDC Center 